\section{(6) OPEN ACCESS}

\title{
Adalimumab long-term safety: infections, vaccination response and pregnancy outcomes in patients with rheumatoid arthritis
}

\author{
Gerd R Burmester, ${ }_{1}^{1}$ Robert Landewé, ${ }^{2}$ Mark C Genovese, ${ }^{3}$ Alan W Friedman, ${ }^{4}$ \\ Nathan D Pfeifer, ${ }^{5}$ Nupun A Varothai, ${ }^{6}$ Ana P Lacerda ${ }^{4}$
}

Handling editor Tore K Kvien

- Additional material is published online only. To view please visit the journal online (http://dx.doi.org/10.1136/ annrheumdis-2016-209322)

${ }^{1}$ Department of Rheumatology and Clinical Immunology, Charité-University Medicine Berlin, Free University and Humboldt University of Berlin, Berlin, Germany ${ }^{2}$ Clinical Immunology and Rheumatology, Academic Medical Centre, University of Amsterdam, Amsterdam, The Netherlands

${ }^{3}$ Division of Immunology and Rheumatology, Stanford University, Palo Alto, California, USA

${ }^{4}$ Immunology Clinical Development, AbbVie, North Chicago, Illinois, USA ${ }^{5}$ Clinical Pharmacology and Pharmacometrics, AbbVie, North Chicago, Illinois, USA ${ }^{6}$ Data and Statistical Sciences, AbbVie, North Chicago, Illinois, USA

\section{Correspondence to}

Dr Gerd R Burmester, Department of Rheumatology and Clinical Immunology, Charité-University Medicine Berlin, Charitéplatz 1, Berlin 10017, Germany; gerd.burmester@charite.de

Received 2 February 2016 Revised 25 April 2016 Accepted 27 May 2016 Published Online First 23 June 2016

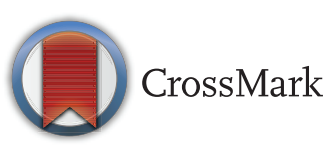

To cite: Burmester GR Landewé $\mathrm{R}$, Genovese MC, et al. Ann Rheum Dis 2017:76:414-417.

\section{ABSTRACT}

Background Adalimumab has been used in patients with moderately to severely active rheumatoid arthritis (RA) for over 10 years and has a well-established safety profile across multiple indications.

Objective To update adverse events (AEs) of special interest from global adalimumab clinical trials in patients with RA.

Methods This analysis includes 15132 patients exposed to adalimumab in global RA clinical trials. AEs of interest included overall infections, laboratory abnormalities and AEs associated with influenza vaccination. Pregnancy outcome data were collected from the Adalimumab Pregnancy Registry.

Results Serious infections and tuberculosis occurred at a rate of 4.7 and 0.3 events/100 patient-years, respectively. Two patients experienced hepatitis $B$ reactivation. No significant laboratory abnormalities were reported with adalimumab-plus-methotrexate compared with placebo-plus-methotrexate. Influenza-related AEs occurred in $5 \%$ of vaccinated patients compared with $14 \%$ of patients not vaccinated during the study. Relative risk of major birth defects and spontaneous abortions in adalimumab-exposed women were similar between that of unexposed women with RA and healthy women.

Conclusions This analysis confirms and expands the known safety profile of adalimumab and reports no additional safety risk of laboratory abnormalities, hepatitis B reactivation and pregnancy outcomes, including spontaneous abortions and birth defects. The benefits of influenza vaccination are reinforced.

Trial registration numbers NCT00195663, NCT00195702, NCT00448383, NCT00049751, NCT00234845, NCT00650390, NCT00235859, NCT00647920, NCT00649545, NCT00647491, NCT00649922, NCT00538902, NCT00420927, NCT00870467, NCT00650156, NCT00647270, NCT01185288, NCT01185301.

\section{INTRODUCTION}

Tumour necrosis factor inhibitors (anti-TNFs) have contributed to improved clinical outcomes in rheumatoid arthritis (RA), but safety has been one point of relative concern. Serious infectious event (SIE) rates are increased in patients using biologics, and risk minimisation activities, such as vaccinations and latent tuberculosis (TB) infection (LTBI) screening and prophylaxis can mitigate infection risk. $^{1}{ }^{2}$ Recently, other adverse events (AEs) have become a focus of interest: viral reactivation, vaccination response, laboratory abnormalities and pregnancy outcomes.

This analysis focuses on these emerging events, using comprehensive data from global adalimumab clinical trials in RA.

\section{PATIENTS AND METHODS}

\section{Data sources}

AE data were derived from 28 global clinical trials (see online supplementary table S1) with adalimumab in patients with RA. The Adalimumab Pregnancy Exposure Registry (APER) is an ongoing prospective, observational, exposure cohort study conducted in North America to monitor pregnancy outcome in women with RA exposed to adalimumab during pregnancy compared with a diseasematched group of women with RA who have not used adalimumab during pregnancy and a cohort of healthy pregnant women. This cohort study is being conducted by the Organization of Teratology Information Specialists (OTIS). ${ }^{3}$ Data reported are collected from 1 February 2004 through 5 November 2013.

\section{Safety assessments and statistical analyses}

The safety population consists of all study patients with any adalimumab use from the first dose through 70 days (five half-lives) after the last study dose. Serious AE (SAE) was defined as fatal or immediately life-threatening; required hospitalisation or prolonged hospitalisation; resulted in persistent or significant disability/incapacity, congenital anomaly or required medical or surgical intervention to prevent a serious outcome. AEs were summarised as events/100 patient-years (E/100 PY) of adalimumab exposure, using preferred terms of the Medical Dictionary for Regulatory Activities (MedDRA) V.15.1 (http://www.meddra.org).

AEs of interest included overall SIEs, TB, opportunistic infections, herpes zoster $(\mathrm{HZ})$ and hepatitis $B$ reactivation.

Percentages of patients who developed anaemia during the first 6 months of adalimumab treatment were determined in two studies in patients with early ( $<1$ year),$^{4} 5$ and one with long-standing RA ( $>1$ year). ${ }^{6}$ Mean change from baseline in fasting serum lipid concentrations (total cholesterol (TC), high-density lipoprotein (HDL) and low-density lipoprotein (LDL)) and haemoglobin ( $\mathrm{Hb})$ were compared between adalimumab-plus-methotrexate 
(MTX) and placebo-plus-MTX (MTX-monotherapy) at week 26 in the OPTIMA study, ${ }^{5}$ using a contrast within a one-way analysis of variance.

Influenza-related AEs rates following influenza vaccination were assessed in adalimumab-treated patients with RA for 10 years. ${ }^{7}$ AEs related to influenza virus infection (within 270 days of vaccination) were tabulated using a predefined MedDRA Query.

All pregnancies enrolled in the OTIS pregnancy registry that reached a pregnancy outcome by November 2013 were included. Pregnancy outcomes included live births, major and minor birth defects, spontaneous abortions, preterm delivery ( $<37$ weeks) and mean gestational age at delivery. Pregnancies exposed to MTX were excluded, due to known teratogenicity. ${ }^{8}$ Maternal characteristics were compared between cohorts using $\chi^{2}$ tests for independence. For reported pregnancy outcomes of live births, major birth defects and $\geq 3$ minor birth defects, a point estimate of the relative risk (RR) and $95 \% \mathrm{CI}$ for the adalimumab-exposed group versus each control group was computed. The primary outcome of major birth defects evaluated all pregnancies ending in at least one live birth, and secondarily all pregnancies with known outcome. Infants with $\geq 3$ minor malformations were evaluated to identify any cases with the same three specific anomalies within each group. To estimate the cumulative probability of spontaneous abortion, only women who enrolled with $<20$ weeks of gestation were used for the Kaplan-Meier (KM) analysis. Cox proportional hazards models were used to estimate the HRs and 95\% CIs.

\section{RESULTS}

\section{Patients}

Through 31 December 2012, adalimumab was administered to 15152 patients in RA studies, representing 24810 PYs of adalimumab exposure. Incidence rates presented in table 1 include rates of both serious and non-serious AEs of interest.

\section{Infections}

SIEs were the most frequently reported SAEs, including pneumonia $(0.7 \mathrm{E} / 100 \mathrm{PYs})$, cellulitis $(0.3 \mathrm{E} / 100 \mathrm{PYs})$, bacterial arthritis and sepsis $(0.2 \mathrm{E} / 100 \mathrm{PYs}$ each). The remaining SIEs were reported at $\leq 0.1 \mathrm{E} / 100$ PYs.

At screening, 1272 (13.5\%) patients were diagnosed with LTBI by a positive tuberculin skin test. During follow-up, 18 patients converted to LTBI status ( $<0.01 \mathrm{E} / 100 \mathrm{PYs})$. Sixty-three active TB events occurred in 59 patients (0.3E/100 PYs).

In all RA studies, 40 opportunistic infections excluding oral candidiasis, $\mathrm{HZ}$ and $\mathrm{TB}$ were reported in 35 patients at a rate of $0.2 \mathrm{E} / 100$ PYs. The most frequently reported opportunistic infections were oesophageal candidiasis $(<0.1 \mathrm{E} / 100 \mathrm{PYs})$ and coccidiomycosis, cytomegalovirus infection and mycobacterium avium complex infection $(<0.1 \mathrm{E} / 100 \mathrm{PYs})$.

\section{Influenza vaccination}

In a post hoc analysis, ${ }^{7}$ vaccine use was evaluated in 553 patients with RA receiving $\geq 1$ dose of adalimumab for up to 10 years (mean 5.6 years). One hundred and eighty patients $(32.5 \%)$ received vaccinations at the discretion of the investigators (average disease duration, 14 years at first vaccination). Of these, $171(30.9 \%)$ received at least one influenza vaccination, with a total of 351 influenza vaccinations administered. Influenza-related AEs occurred in 5\% (8/171) of vaccinated patients compared with $14 \%(55 / 382)$ of patients not vaccinated during the study (see online supplementary figure S2).
Table 1 Incidence rates of adverse events of interest in patients with rheumatoid arthritis treated with adalimumab $(\mathrm{N}=15$ 152; 24,810.4 PYs)

\begin{tabular}{|c|c|}
\hline Adverse events & $\mathrm{E}$ (E/100 PYs) \\
\hline Serious infections & $1154(4.7)$ \\
\hline Active tuberculosis (TB) & $63(0.3)$ \\
\hline Opportunistic & $14(<0.1)$ \\
\hline Zoster & $19(<0.1)$ \\
\hline \multicolumn{2}{|l|}{ Non-serious infections } \\
\hline $\begin{array}{l}\text { Opportunistic infections, excluding oral candidiasis, } \\
\text { herpes zoster and TB }\end{array}$ & $26(0.1)$ \\
\hline Herpes zoster & $424(1.7)$ \\
\hline Reactivation of hepatitis $B^{*}$ & $3(<0.1)$ \\
\hline
\end{tabular}

${ }^{*}$ Two patients experienced three events of hepatitis B reactivation: chronic hepatitis $B$, hepatitis B and viral hepatitis carrier.

$E$, number of events; E/100 PYs, events per 100 patient-years.

\section{Laboratory parameters \\ Haematology}

After 6 months of treatment, incidence of moderate anaemia $(\mathrm{Hb} 8.0$ to $<10 \mathrm{~g} / \mathrm{L}$ ) was significantly less in patients treated with adalimumab-plus-MTX for both early $(\mathrm{p}<0.05)$ and longstanding RA $(\mathrm{p}<0.05)$. Mean increases in Hb levels was higher in patients treated with adalimumab-plus-MTX in both early $(\mathrm{p}<0.05)$ and long-standing RA $(\mathrm{p}<0.05)$ (table 2).

\section{Metabolic}

At baseline, mean fasting TC, LDL and HDL were $4.974 \mathrm{mmol} /$ $\mathrm{L}(192 \mathrm{mg} / \mathrm{dL}), 2.851 \mathrm{mmol} / \mathrm{L}(110 \mathrm{mg} / \mathrm{dL})$ and $1.423 \mathrm{mmol} / \mathrm{L}$ $(55 \mathrm{mg} / \mathrm{dL})$, respectively, in the adalimumab-plus-MTX treatment group and $5.337 \mathrm{mmol} / \mathrm{L}(206 \mathrm{mg} / \mathrm{dL}), 3.146 \mathrm{mmol} / \mathrm{L}$ $(121 \mathrm{mg} / \mathrm{dL})$ and $1.562 \mathrm{mmol} / \mathrm{L}(60 \mathrm{mg} / \mathrm{dL})$, respectively, in the MTX-monotherapy group. After 6 months, the mean changes in TC and LDL were higher in patients treated with adalimumab-plus-MTX compared with that of MTX-monotherapy (see online supplementary figure S1). Mean HDL changes were not different in the adalimumab-plus-MTX group compared with that of MTX-monotherapy. The increases in TC and LDL concentrations in both groups were mild and not clinically significant. At baseline, mean Apo A-1, Apo B and lipoprotein were 131,81 and $20 \mathrm{mg} / \mathrm{dL}$, respectively, in the adalimumabplus-MTX group and 131,81 and $21 \mathrm{mg} / \mathrm{dL}$, respectively, in the MTX-monotherapy group. Mean Apo A-1, Apo B and lipoprotein changes were not different in the adalimumab-plus-MTX group compared with that of MTX-monotherapy.

\section{Exposure during pregnancy}

A total of 154 women (74 exposed to adalimumab, 80 with RA not exposed to adalimumab) enrolled in the OTIS registry. The mean gestational age at delivery of live births was similar between the two groups ( 38.5 vs 38.2 weeks, $p=0.30$ ). Of 74 pregnant women exposed to adalimumab, approximately $40 \%$, $16 \%$ and $44 \%$ used adalimumab in the first trimester only, for two trimesters and throughout pregnancy, respectively. Per protocol, MTX was not allowed to be used concomitantly with adalimumab in the OTIS pregnancy registry. However, there were five patients using adalimumab-plus-methotrexate at inclusion into OTIS registry and none on MTX in the RA control population.

The frequencies and RR of major birth defects (table 3) were not different between adalimumab-exposed women, unexposed 
Table 2 Frequency and mean change from baseline in $\mathrm{Hb}$ values at 6 months of treatment in two studies in patients with early RA, ${ }^{4}$ and one in long-standing patients with $\mathrm{RA}^{6}$

\begin{tabular}{|c|c|c|c|c|}
\hline & \multicolumn{2}{|l|}{ Early RA } & \multicolumn{2}{|l|}{ Long-standing RA } \\
\hline & $\begin{array}{l}\text { Placebo-plus-MTX } \\
\mathrm{N}=774\end{array}$ & $\begin{array}{l}\text { Adalimumab-plus-MTX } \\
\mathrm{N}=783\end{array}$ & $\begin{array}{l}\text { Placebo-plus-MTX } \\
\mathrm{N}=200\end{array}$ & $\begin{array}{l}\text { Adalimumab-plus-MTX } \\
\mathrm{N}=207\end{array}$ \\
\hline \multicolumn{5}{|l|}{ Anaemia } \\
\hline $\mathrm{Hb}<10.0-8.0 \mathrm{~g} / \mathrm{dL}$ & $55(7.1)$ & $28(3.6)^{*}$ & $11(5.5)$ & $3(1.4)^{*}$ \\
\hline $\mathrm{Hb}<8.0-6.5 \mathrm{~g} / \mathrm{dL}$ & $4(0.5)$ & $2(0.3)$ & 0 & 0 \\
\hline \multicolumn{5}{|l|}{$\mathrm{Hb}$ decreased } \\
\hline $\mathrm{Hb}$, mean change $(\mathrm{g} / \mathrm{dL})$ & -0.24 & $+0.45^{*}$ & -0.18 & $+0.44^{*}$ \\
\hline
\end{tabular}

Values are listed as $\mathrm{n}(\%)$ unless otherwise indicated.

${ }^{*} p<0.05$ for adalimumab-plus-MTX versus MTX-monotherapy. $\chi^{2}$ test for frequency rates.

$\mathrm{Hb}$, haemoglobin; MTX, methotrexate; RA, rheumatoid arthritis.

\begin{tabular}{|c|c|c|c|c|c|}
\hline & $\begin{array}{l}\text { Adalimumab-exposed cohort } \\
\mathrm{N}=74 \\
\mathrm{n}(\%)\end{array}$ & $\begin{array}{l}\text { RA Comparison cohort } \\
\mathrm{N}=80 \\
\mathrm{n}(\%)\end{array}$ & RR $(95 \% \mathrm{Cl})$ & $\begin{array}{l}\text { Healthy cohort } \\
\mathrm{N}=219 \\
\mathrm{n}(\%)\end{array}$ & RR $(95 \% \mathrm{Cl})$ \\
\hline Live births & $65(87.8)$ & $74(92.5)$ & $0.95(0.85$ to 1.05$)$ & $198(90.4)$ & 0.97 (0.88 to 1.07$)$ \\
\hline Major birth defects among live births & $3 / 65(4.6)$ & $4 / 74(5.4)$ & $0.75^{*}$ (0.13 to 3.61$)$ & 10/198 (5.1) & 0.91 (0.26 to 3.22$)$ \\
\hline Major birth defects among all pregnancies & $3 / 72(4.2)$ & $5 / 77(6.5)$ & $0.72 *(0.14$ to 3.50$)$ & $10 / 202(5.0)$ & 0.77 (0.22 to 2.67$)$ \\
\hline$\geq 3$ Minor malformations $\dagger$ & $12 / 45(26.7)$ & $18 / 64(28.1)$ & $1.15 \ddagger(0.43$ to 3.08$)$ & $31 / 123(25.2)$ & $1.06 \S(0.41$ to 2.53$)$ \\
\hline
\end{tabular}

*Adjusted for RA disease severity (impairment) measure; adjusted OR and $95 \% \mathrm{Cl}$ is reported as an estimate of the adjusted $\mathrm{RR}$ and $95 \% \mathrm{Cl}$. †Reflects the number with pattern of minor malformations among infants receiving dysmorphological examination.

¥Adjusted for maternal education, RA disease severity measures (impairment, pain) and sex of infant; adjusted OR and $95 \% \mathrm{Cl}$ are reported as estimates of the adjusted RR and $95 \% \mathrm{Cl}$. $\S$ Adjusted for prednisone use; adjusted OR and $95 \% \mathrm{Cl}$ are reported as estimates of the adjusted RR and $95 \% \mathrm{Cl}$.

$R A$, rheumatoid arthritis; RR, relative risk.

women with RA and healthy women. Among the specific birth defects in the adalimumab cohort, no pattern was evident. Specific major birth defects in the adalimumab-exposed cohort were ventricular septal defect (resolved), microcephaly and undescended testicle. In the RA cohort, ventricular septal defect (resolved), microcephaly, talipes equinovarus, cataract (not otherwise specified (NOS)) and chromosomal anomaly NOS were observed. Likewise, rates of $\geq 3$ minor birth defects were similar among the three cohorts, with no two infants in the adalimumab-exposed group having the same three specific minor anomalies.

The adjusted HR for spontaneous abortion in the adalimumabexposed cohort relative to the RA comparison group was not significantly elevated (adjusted HR $=2.06,95 \%$ CI 0.53 to 7.98); however, the number of events was small $(n=10)$. No stillbirths were reported in the adalimumab-exposed cohort. Preterm delivery occurred at similar rates between the adalimumab-exposed and RA comparison groups (adjusted $\mathrm{HR}=1.08$, 95\% CI 0.41 to 2.83).

\section{DISCUSSION}

Anti-TNF therapy is associated with an increased risk of infections. The spectrum of infections reported herein is similar to those reported with other anti-TNFs in both randomised clinical trials and registries, ${ }^{9-12}$ and the types of infections have not changed over time. ${ }^{2} 13$

Opportunistic infections, although uncommon, may be challenging from a diagnostic perspective, given the variety of possible bacterial, mycobacterial, fungal, viral and parasitic agents.
Recently, there has been great interest in $\mathrm{HZ} .{ }^{14}$ The overall rate with adalimumab was low and similar to reported rates in registries and a large claims database. ${ }^{14-16}$ This suggests that the use of anti-TNF therapies may not put patients at higher risk of $\mathrm{HZ}$, but the risk:benefit for vaccination prior to initiating biological therapies remains compelling.

Overall, the effect of adalimumab on $\mathrm{Hb}$ and lipid parameters suggests no new safety signals in relation to MTX-monotherapy, in both early and long-standing RA. ${ }^{17}$ Monitoring lipid changes is of particular importance since risk of cardiovascular events may be increased in RA.

Routine vaccination of patients with RA with non-live agents, even in the setting of ongoing anti-TNF treatment, is recommended by the Advisory Committee on Immunization Practices. ${ }^{1}$ However, awareness of this appears to be low, since a minority of patients with RA starting adalimumab in our studies received influenza vaccination. Patients that received this vaccine clearly presented with fewer influenza-related AEs over an average of 5.6 years of follow-up, compared with patients not receiving the vaccine.

Like other anti-TNFs, adalimumab is classified as pregnancy category B (no documented human toxicity) by the US Food and Drug Administration. A reproductive animal study revealed no evidence of fetal harm due to adalimumab, ${ }^{18}$ but only a small number of adalimumab-exposed human pregnancies have been published to date. ${ }^{19-21}$ The OTIS registry reported neither specific pattern of defects in adalimumab-exposed infants during prenatal development nor adverse pregnancy outcomes. 


\section{Strengths and limitations}

Strengths of this adalimumab safety analysis include the number of patients, the duration of adalimumab exposure and the close monitoring in clinical trials compared with postmarketing surveillance or non-interventional studies. Our focus was on safety aspects that have not been broadly published with adalimumab or other anti-TNFs.

Limiting this analysis to clinical trial experience may not fully reflect clinical practice, due to selection bias of trial populations, survival of completers and comorbidities. Comparisons with other treatments are limited due to the lack of a control group with any specific active treatment.

\section{CONCLUSIONS}

The analysis of this large clinical trial database of patients with moderate to severe RA updates and confirms the known safety profile of adalimumab with regard to infections, laboratory abnormalities, hepatitis $\mathrm{B}$ reactivation and adverse pregnancy outcomes. Finally, the benefit of influenza vaccination for patients with RA is reinforced.

Acknowledgements AbbVie funded the studies, contributed to the design, was involved in the collection, analysis and interpretation of the data, and in the writing, review and approval of the publication. Medical writing support was provided by Kathleen V. Kastenholz, PharmD, MS and Gaurav Patki, PhD of AbbVie.

Contributors GRB, RL, MCG, AWF and APL contributed to the design, review and interpretation of the data. NDP contributed to the review and interpretation of the data. NAV contributed to the analysis of the data.

Funding AbbVie sponsored the clinical trials.

Competing interests GRB has received research grants, consulting fees and speaker's fees from AbbVie, BMS, Merck, Pfizer, Roche and UCB. RL has received consulting fees from AbbVie, Amgen, BMS, Centocor, GSK, Merck, Novartis, Pfizer, Roche, Schering-Plough, UCB and Wyeth and is owner of Rheumatology Consultancy BV. MCG has received research grants and consulting fees from AbbVie. AWF, NDP and APL are employees of AbbVie and may hold stock and/or options. NV is a former employee of AbbVie and may hold stock and/or options.

Patient consent Obtained.

Ethics approval Appropriate Ethics Committee/Institutional review board approval was obtained.

Provenance and peer review Not commissioned; externally peer reviewed.

Open Access This is an Open Access article distributed in accordance with the Creative Commons Attribution Non Commercial (CC BY-NC 4.0) license, which permits others to distribute, remix, adapt, build upon this work non-commercially, and license their derivative works on different terms, provided the original work is properly cited and the use is non-commercial. See: http://creativecommons.org/ licenses/by-nc/4.0/

\section{REFERENCES}

1 National Center for, Immunization Respiratory, Diseases. General recommendations on immunization-recommendations of the Advisory Committee on Immunization Practices (ACIP). MMWR Recomm Rep 2011;60:1-64.

2 Schiff MH, Burmester GR, Kent JD, et al. Safety analyses of adalimumab (HUMIRA) in global clinical trials and US postmarketing surveillance of patients with rheumatoid arthritis. Ann Rheum Dis 2006;65:889-94.
3 Briggs GG, Polifka J, Research Committee, Organization of Teratology Information Specialists. Better data needed from pregnancy registries. Birth Defects Res Part A Clin Mol Teratol 2009;85:109-11.

4 Breedveld FC, Weisman MH, Kavanaugh AF, et al. The PREMIER study: a multicenter, randomized, double-blind clinical trial of combination therapy with adalimumab plus methotrexate versus methotrexate alone or adalimumab alone in patients with early, aggressive rheumatoid arthritis who had not had previous methotrexate treatment. Arthritis Rheum 2006:54:26-37.

5 Kavanaugh A, Fleischmann RM, Emery P, et al. Clinical, functional and radiographic consequences of achieving stable low disease activity and remission with adalimumab plus methotrexate or methotrexate alone in early rheumatoid arthritis: 26-week results from the randomised, controlled OPTIMA study. Ann Rheum Dis 2013:72:64-71.

6 Keystone EC, Kavanaugh AF, Sharp JT, et al. Radiographic, clinical, and functional outcomes of treatment with adalimumab (a human anti-tumor necrosis factor monoclonal antibody) in patients with active rheumatoid arthritis receiving concomitant methotrexate therapy: a randomized, placebo-controlled, 52-week trial. Arthritis Rheum 2004;50:1400-11.

7 Keystone EC, van der Heijde D, Kavanaugh A, et al. Clinical, functional, and radiographic benefits of longterm adalimumab plus methotrexate: final 10-year data in longstanding rheumatoid arthritis. J Rheumatol 2013;40:1487-97.

8 Hyoun SC, Običan SG, Scialli AR. Teratogen update: methotrexate. Birth Defects Res Part A Clin Mol Teratol 2012;94:187-207.

9 Galloway JB, Hyrich KL, Mercer LK, et al. Anti-TNF therapy is associated with an increased risk of serious infections in patients with rheumatoid arthritis especially in the first 6 months of treatment: updated results from the British Society for Rheumatology Biologics Register with special emphasis on risks in the elderly. Rheumatology (Oxford) 2011:50:124-31.

10 Kay J, Fleischmann R, Keystone E, et al. Golimumab 3-year safety update: an analysis of pooled data from the long-term extensions of randomised, double-blind, placebo-controlled trials conducted in patients with rheumatoid arthritis, psoriatic arthritis or ankylosing spondylitis. Ann Rheum Dis 2015;74:538-46.

11 Listing J, Strangfeld A, Kary S, et al. Infections in patients with rheumatoid arthritis treated with biologic agents. Arthritis Rheum 2005;52:3403-12.

12 Weinblatt ME, Bathon JM, Kremer JM, et al. Safety and efficacy of etanercept beyond 10 years of therapy in North American patients with early and longstanding rheumatoid arthritis. Arthritis Care Res (Hoboken) 2011;63:373-82.

13 Burmester GR, Panaccione R, Gordon KB, et al. Adalimumab: long-term safety in 23458 patients from global clinical trials in rheumatoid arthritis, juvenile idiopathic arthritis, ankylosing spondylitis, psoriatic arthritis, psoriasis and Crohn's disease. Ann Rheum Dis 2013;72:517-24.

14 Winthrop KL, Baddley JW, Chen L, et al. Association between the initiation of anti-tumor necrosis factor therapy and the risk of herpes zoster. JAMA 2013:309:887-95.

15 Galloway JB, Mercer LK, Moseley A, et al. Risk of skin and soft tissue infections (including shingles) in patients exposed to anti-tumour necrosis factor therapy: results from the British Society for Rheumatology Biologics Register. Ann Rheum Dis 2013;72:229-34

16 Zisman D, Bitterman $\mathrm{H}$, Shalom $\mathrm{G}$, et al. Psoriatic arthritis treatment and the risk of herpes zoster. Ann Rheum Dis 2016;75:131-5.

17 Burmester GR, Kivitz AJ, Kupper H, et al. Efficacy and safety of ascending methotrexate dose in combination with adalimumab: the randomised CONCERTO trial. Ann Rheum Dis 2015;74:1037-44.

18 Humira [package insert]. North Chicago, IL, USA: AbbVie, 2014.

19 Berthelot JM, De Bandt M, Goupille P, et al. Exposition to anti-TNF drugs during pregnancy: outcome of 15 cases and review of the literature. Joint Bone Spine 2009:76:28-34.

20 Diav-Citrin 0, Otcheretianski-Volodarsky A, Shechtman S, et al. Pregnancy outcome following gestational exposure to TNF-alpha-inhibitors: a prospective, comparative, observational study. Reprod Toxicol 2014;43:78-84.

21 Roux $\mathrm{CH}$, Brocq $\mathrm{O}$, Breuil $\mathrm{V}$, et al. Pregnancy in rheumatology patients exposed to anti-tumour necrosis factor (TNF)-alpha therapy. Rheumatology (Oxford) 2007:46:695-8. 